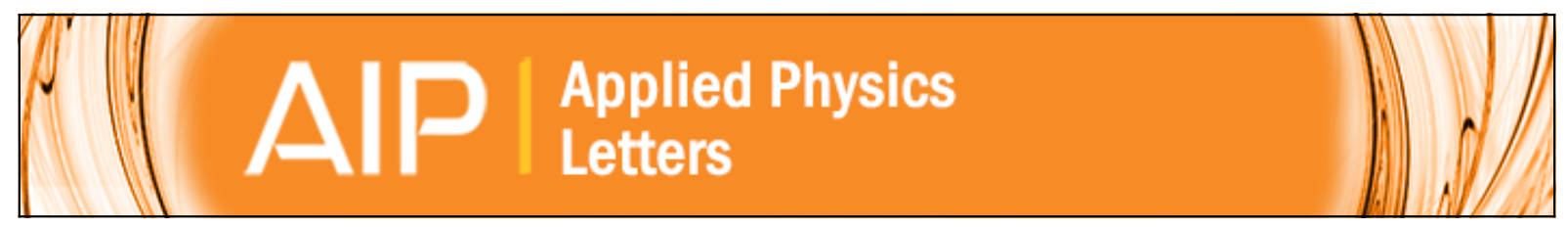

\title{
A resonant spin lifetime transistor
}

X. Cartoixà, D. Z.-Y. Ting, and Y.-C. Chang

Citation: Applied Physics Letters 83, 1462 (2003); doi: 10.1063/1.1601693

View online: http://dx.doi.org/10.1063/1.1601693

View Table of Contents: http://scitation.aip.org/content/aip/journal/apl/83/7?ver=pdfcov

Published by the AIP Publishing

\section{AlP Re-register for Table of Content Alerts}

\section{Create a profile. \\ Sign up today!}




\title{
A resonant spin lifetime transistor
}

\author{
X. Cartoixàa) \\ Department of Physics, University of Illinois at Urbana-Champaign, Urbana, Illinois 61801 \\ D. Z.-Y. Ting \\ Jet Propulsion Laboratory, California Institute of Technology, Pasadena, California 91109 \\ Y.-C. Chang \\ Department of Physics, University of Illinois at Urbana-Champaign, Urbana, Illinois 61801
}

(Received 15 April 2003; accepted 28 May 2003)

\begin{abstract}
We present a device concept for a spintronic transistor based on the spin relaxation properties a two-dimensional electron gas (2DEG). The device design is very similar to that of the Datta and Das spin transistor. However, our proposed device works in the diffusive regime rather than in the ballistic regime. This eases lithographical and processing requirements. The switching action is achieved through the biasing of a gate contact, which controls the lifetime of spins injected into the 2DEG from a ferromagnetic emitter, thus allowing the traveling spins to be either aligned with a ferromagnetic collector or randomizing them before collection. The device configuration can easily be turned into a memory and a readout head for magnetically stored information. (C) 2003 American Institute of Physics. [DOI: 10.1063/1.1601693]
\end{abstract}

If the current pace of electronic device miniaturization is to continue, it is reasonable to think that the good use of the quantum properties of the electron will play a role in making this possible. Traditionally it has been the wave character of the electron that has been put to this use, resulting in devices such as the resonant tunnel diode $^{1}$ and the single electron transistor. ${ }^{2}$ Another quantum property of the electron that only recently has received attention for its potential for information storage and processing is its spin. One of the most promising proposals for a spin electronic (spintronic) device is the spin transistor by Datta and Das. ${ }^{3}$ In that device, electrons are injected from a ferromagnetic contact into a twodimensional electron gas (2DEG) and another ferromagnetic contact is used to collect the traveling electrons and analyze their spin. Switching action is achieved by the application of an external electric field, which affects the spin of the ensemble through the Rashba ${ }^{4}$ effect.

In this letter we see how, by making minimal changes to the device configuration proposed by Datta and Das, and by considering the bulk inversion asymmetry ${ }^{5}$ (BIA) effects, we can create a family of information processing, storing, and retrieving devices. We start reviewing the effects in the 2DEG band structure and spin alignments of the combination of BIA and structural inversion asymmetry (SIA), and the consequences for the spin lifetime of the electrons in the 2DEG. Then, we describe our proposal for a spin transistor and make some estimates of its performance. Finally, we also describe two kinds of memory cells and a read head based on these effects.

For zinc-blende semiconductor heterostructures we can easily write a Hamiltonian describing both the SIA $^{4}$ and $\mathrm{BIA}^{6,7}$ contributions to the 2DEG lowest conduction subbands of a [001]-grown structure

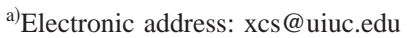

$$
\begin{aligned}
H & =H_{0}+\alpha_{\mathrm{BIA}}\left(\sigma_{x} k_{x}-\sigma_{y} k_{y}\right)+\alpha_{\mathrm{SIA}}\left(\sigma_{x} k_{y}-\sigma_{y} k_{x}\right) \\
& =H+\sigma_{x}\left(\alpha_{\mathrm{SIA}} k_{y}+\alpha_{\mathrm{BIA}} k_{x}\right)-\sigma_{y}\left(\alpha_{\mathrm{BIA}} k_{y}+\alpha_{\mathrm{SIA}} k_{x}\right),
\end{aligned}
$$

where $H_{0}$ is the spin degenerate part of the Hamiltonian, $\alpha_{\text {BIA }}\left(\alpha_{\text {SIA }}\right)$ parameterizes the importance of the BIA (SIA) contribution- $\alpha_{\text {SIA }}$ is commonly referred to as the Rashba coefficient - to the lowest nonvanishing order, $\sigma_{i}$ are the Pauli matrices and $k_{i}$ are the components of the in-plane electron wave vector $\mathbf{k}$.

For our forthcoming considerations it is important to study the spin configuration in $\mathbf{k}$ space that results from Eq. (1). When $\alpha_{\mathrm{BIA}}=0$, the electron spins point in the 2DEG plane and perpendicular to $\mathbf{k}$ (see, for example, Fig. 1 in Ref. 8). Figure 1 shows the spin expectation values for electrons with wavevector in the 2DEG plane and a magnitude $k$ $=0.012 \pi / a$. The expectation values have been calculated from the eigenstates of Hamiltonian (1). For $\alpha_{\mathrm{BIA}} / \alpha_{\mathrm{SIA}}=0.25$ we see that the spins are slightly tilted respect to the tangential configuration for the case of $\alpha_{\text {BIA }}=0$. However, the usual time reversal requirement that states
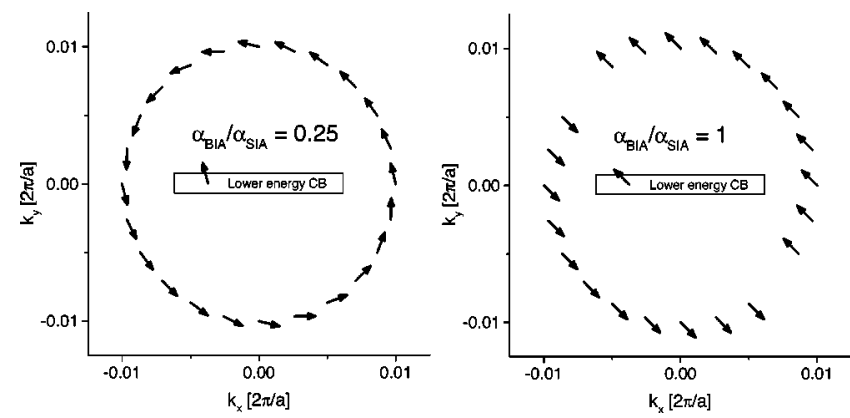

FIG. 1. Spin directions for the lower conduction subband of a 2DEG. The spins are plotted at $15^{\circ}$ intervals, and correspond to states lying on a circle in the $k_{x}-k_{y}$ plane with $k=0.01 \AA$. The two plots are for different relative contributions of BIA and SIA. Note the configuration for the case $\alpha_{\text {SIA }}$ $=\alpha_{\mathrm{BIA}}$. In that case, no spin direction is specified for the [1 $\left.\overline{10}\right]$ and $[\overline{1} 10]$ directions because the states are spin degenerate. 


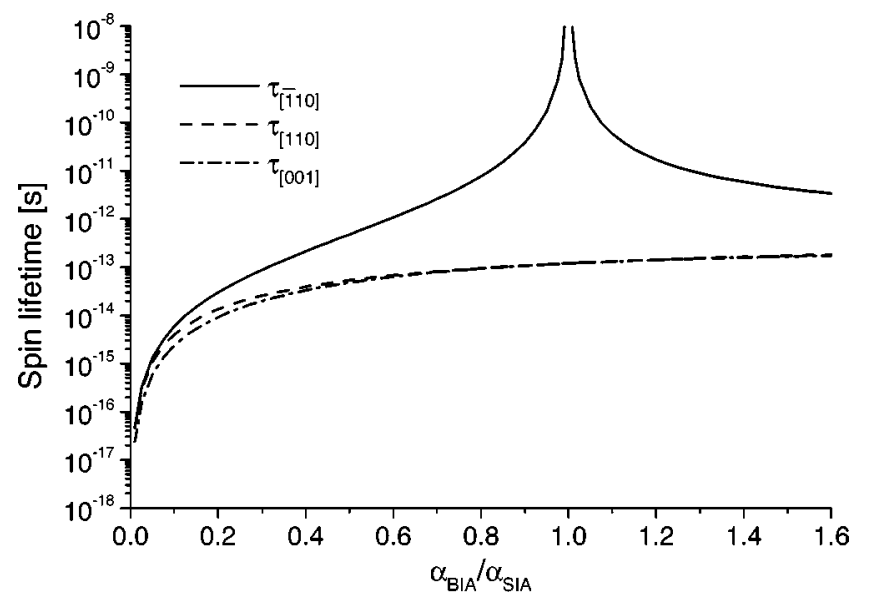

FIG. 2. The three diagonal elements of the spin lifetime tensor as a function of the ratio of the BIA and the SIA parameters.

within a subband with opposite $\mathbf{k}$ have opposite spin is kept. This ensures that, in this single electron picture, there is no magnetism in the 2 DEG.

The spin configuration in Fig. 1 for $\alpha_{\mathrm{BIA}} / \alpha_{\mathrm{SIA}}=1$ is much more interesting. In it, eigenstate spins can only point in one of two possible directions, namely [1 $\overline{1} 0]$ and [ $\overline{1} 10]$, depending on the angle $\theta$ between $\mathbf{k}$ and the [100] direction. ${ }^{9}$ The boundary of the two regions is at $\theta=-\pi / 4$ and $\theta=3 \pi / 4$. The condition $\alpha_{\text {BIA }}=\alpha_{\text {SIA }}$ might be achieved by tuning the value of $\alpha_{\text {SIA }}$ through the application of an external electric field perpendicular to the 2DEG. ${ }^{10}$ The value of $\alpha_{\mathrm{BIA}}$ is primarily determined by the intrinsic properties of the constituent materials, and its magnitude can only be affected by the thickness of the $2 \mathrm{DEG}^{6}$

In this particular configuration, the component along [110] of an injected spin will always be aligned with the effective magnetic field ${ }^{7}$ irrespective of $\mathbf{k}$ and, therefore, will not suffer from the different rates and axes of precession as described by the D'yakonov-Perel'11 (DP) mechanism of spin relaxation. Averkiev and Golub ${ }^{12,13}$ have computed the DP spin lifetimes of the different components for electrons in the [001] 2DEG. In particular, assuming the validity of Eq. (1), they see that the lifetime for spins along [ $\overline{1} 10]$ is proportional to $\left(\alpha_{\mathrm{SIA}}-\alpha_{\mathrm{BIA}}\right)^{-2}$. Figure 2 shows the spin lifetimes plotted from Eq. (19) in Ref. 13, where $\tau_{i}$ is the lifetime of the spin component along $i$. The values used in the computation are $k_{F}=0.01 \AA^{-1}$ (a degenerate 2DEG is assumed), a momentum scattering time $\tau_{p}=0.2 \mathrm{ps}$ and $\alpha_{\text {BIA }}$ $=15 \times 10^{-10} \mathrm{eV} \mathrm{cm} .{ }^{14}$ From this figure, we see clearly the resonant behavior of the lifetime when $\alpha_{\text {SIA }}=\alpha_{\text {BIA }}$. In what follows, we will describe how, by driving this component of the spin in and out of resonance through the action of an external bias, we can construct a series of spintronic devices.

Figure 3 shows the operating principle of the resonant spin lifetime transistor (RSLT). The device layout is very similar to the Datta-Das ${ }^{3}$ device, except that the ferromagnetic contacts must be designed so that their magnetization points in the $[\overline{1} 10]$ direction, as per Fig. 1. Since the resonant spin direction points in the 2DEG plane, this may be easily achieved with a thin bar as in the figure. At first, an ensemble of spins pointing along $[\overline{1} 10]$ is injected in the 2DEG. The gate bias drives the lifetime of the injected spins on- or off-

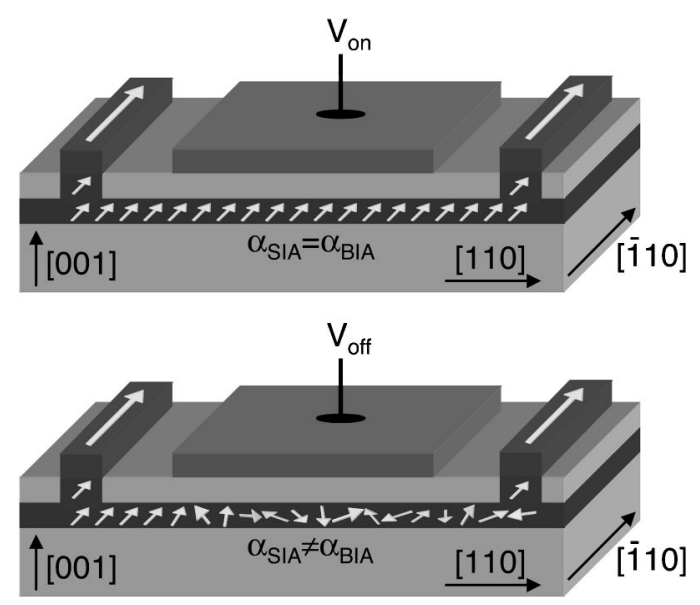

FIG. 3. Operating principle of the resonant spin lifetime transistor. The gate bias drives the lifetime of the injected spins on- or off-resonance. In the on state the spins would arrive aligned with the ferromagnetic collector, thus resulting in low resistance. In the off state, the spins are randomized before reaching the collector and a high resistance is measured.

resonance by setting $\alpha_{\mathrm{BIA}}=\alpha_{\mathrm{SIA}}$ or $\alpha_{\mathrm{BIA}} \neq \alpha_{\mathrm{SIA}}$, respectively. In the on state the spins would arrive aligned with the ferromagnetic collector, thus resulting in low resistance. In the off state, the spins are randomized before reaching the collector and a high resistance is measured.

This operating mechanism indicates some of the design considerations that the device must meet. Since its operation is based on the control of the DP relaxation mechanism, the injected electrons must undergo several momentum scattering events for proper functioning. This implies that the device must operate in the drift-diffusive regime. Thus, the fabrication requirements are less demanding than for the Datta-Das device, ${ }^{3}$ which operates in the ballistic regime. ${ }^{15}$ Of course, the resonant spin lifetime will have a finite magnitude, due to either other relaxation mechanisms ${ }^{16}$ or to higher order terms that have been neglected in the Hamiltonian. This finite lifetime in the on mode limits the maximum transit times the design can sustain. We can get an estimate of these lifetimes from measurements in [110]grown quantum wells. ${ }^{16}$ There, the DP mechanism for spins perpendicular to the well is suppressed, and the resulting lifetime is increased to several nanoseconds.

The turn off time will be governed by the spin lifetime of the electrons in the nonresonant condition. This is a short time, of the order of a few picoseconds. ${ }^{17}$ The turn on time is given by how fast the random spins can be evacuated from the channel and substituted for correctly aligned spins, which is again estimated by the transit time in the channel. Transit times of a few picoseconds or less ${ }^{18}$ are achievable in the operation of high electron mobility transistors. Thus, one would expect operating frequencies of the order of a few hundreds of gigahertz; the limiting factor being the turn-on time. Of course, if a reduction of the transit time is pursued, care must be taken to ensure that the RSLT still operates in the drift-diffusive regime. An experimental demonstration of this device would be free from considerations of local Hall effect $^{19}$ because the orientation of the magnetizations would not be reversed.

There are other kinds of devices that can be constructed with these building blocks. If the gate bias in Fig. 3 is ap- 

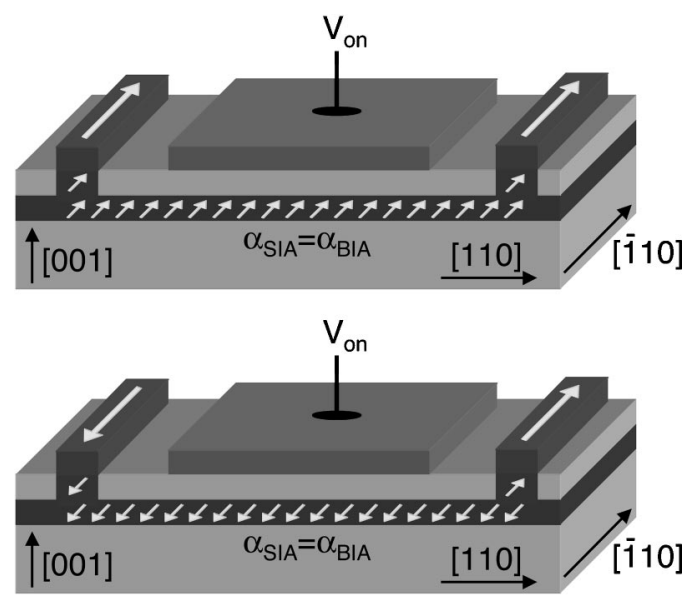

FIG. 4. Operating principle of the resonant spin lifetime memory. The gate bias is fixed in the resonant condition. Different memory states are stored by the relative orientation of the emitter and collector magnetizations. The reading of the state is obtained by the application of a current and measurement of the resistance.

plied through a charged/uncharged floating gate, the device would behave as a flash memory. A different nonvolatile memory configuration is shown in Fig. 4. In it, the gate bias is set to the resonance condition only during the read cycle and remains off at other times, thus reducing power consumption. Then, the " 0 " or " 1 " states would be given by the relative orientation of the magnetization of the emitter and the collector. The performance would improve because this memory can operate in the ballistic mode. Finally, we can envision a magnetic information readout head based on the configuration in Fig. 4. Similar to giant magnetoresistance readout heads, ${ }^{20}$ the magnetization of one contact would be pinned while the other follows some stored pattern.

Note added in proof. We have recently become aware of a similar work by Schliemann, Egues and Loss. ${ }^{21}$ Their work emphasizes the treatment of quantum point contacts, which have different implications for the collection of spins from the extended contacts we consider in this work.

The authors thank Dr. Stuart Wolf for helpful discussions. This work was supported in part by Defense Advanced Research Projects Agency (DARPA) under Contract No. DAAD19-01-1-0324. A part of this work was carried out at the Jet Propulsion Laboratory, California Institute of Technology, and was sponsored by the DARPA-SpinS program through an agreement with the National Aeronautics and Space Administration.

${ }^{1}$ L. Esaki and R. Tsu, IBM J. Res. Dev. 14, 61 (1970).

${ }^{2}$ K. K. Likharev, Proc. IEEE 87, 606 (1999), and references therein.

${ }^{3}$ S. Datta and B. Das, Appl. Phys. Lett. 56, 665 (1990).

${ }^{4}$ Y. A. Bychkov and E. I. Rashba, J. Phys. C 17, 6039 (1984).

${ }^{5}$ G. Dresselhaus, Phys. Rev. 100, 580 (1955).

${ }^{6}$ R. Eppenga and M. F. H. Schuurmans, Phys. Rev. B 37, 10923 (1988).

${ }^{7}$ E. A. D. E. Silva, Phys. Rev. B 46, 1921 (1992).

${ }^{8}$ T. Schäpers, G. Engels, J. Lange, T. Klocke, M. Hollfelder, and H. Lüth, J. Appl. Phys. 83, 4324 (1998).

${ }^{9}$ D. Z.-Y. Ting and X. Cartoixà, Phys. Rev. B (submitted).

${ }^{10}$ J. Nitta, T. Akazaki, H. Takayanagi, and T. Enoki, Phys. Rev. Lett. 78, 1335 (1997)

${ }^{11}$ M. I. D'yakonov and V. I. Perel', JETP Lett. 13, 467 (1971).

${ }^{12}$ N. S. Averkiev and L. E. Golub, Phys. Rev. B 60, 15582 (1999).

${ }^{13}$ N. S. Averkiev, L. E. Golub, and M. Willander, J. Phys.: Condens. Matter 14, R271 (2002).

${ }^{14}$ X. Cartoixà, D. Z.-Y. Ting, and T. C. McGill, cond-mat/0212394 (2002), Phys. Rev. B (submitted).

${ }^{15}$ H. X. Tang, F. G. Monzon, R. Lifshitz, M. C. Cross, and M. L. Roukes, Phys. Rev. B 61, 4437 (2000).

${ }^{16}$ Y. Ohno, R. Terauchi, T. Adachi, F. Matsukura, and H. Ohno, Phys. Rev. Lett. 83, 4196 (1999).

${ }^{17}$ A. Tackeuchi, Y. Nishikawa, and O. Wada, Appl. Phys. Lett. 68, 797 (1996).

${ }^{18}$ K. Shinohara, Y. Yamashita, A. Endoh, K. Hikosaka, T. Matsui, T. Mimura, and S. Hiyamizu, IEEE Electron Device Lett. 22, 507 (2001).

${ }^{19}$ F. G. Monzon and M. L. Roukes, J. Magn. Magn. Mater. 199, 632 (1999).

${ }^{20}$ C. Tsang, R. E. Fontana, T. Lin, D. E. Heim, V. S. Speriosu, B. A. Gurney, and M. L. Mason, IEEE Trans. Magn. 30, 3801 (1994).

${ }^{21}$ J. Schliemann, J. C. Egues, and D. Loss, Phys. Rev. Lett. 90, 146801 (2003). 